\title{
Un théâtre qui exalte le vivre : Nathalie Papin, Dominique Richard, Betty Heurtebise, Joël \\ Pommerat
}

Theatre Glorifying Life: Nathalie Papin, Dominique Richard, Betty Heurtebise, Joël Pommerat

Aurélie Armellini

\section{(2) OpenEdition}

\section{Journals}

Édition électronique

URL : https://journals.openedition.org/recherchestravaux/775

DOI : 10.4000/recherchestravaux.775

ISSN : 1969-6434

Éditeur

UGA Éditions/Université Grenoble Alpes

Édition imprimée

Date de publication : 1 décembre 2015

Pagination : $39-49$

ISBN : $978-2-84310-314-8$

ISSN : 0151-1874

\section{Référence électronique}

Aurélie Armellini, « Un théâtre qui exalte le vivre : Nathalie Papin, Dominique Richard, Betty Heurtebise, Joël Pommerat », Recherches \& Travaux [En ligne], 87 | 2015, mis en ligne le 01 janvier 2017, consulté le 29 octobre 2021. URL : http://journals.openedition.org/recherchestravaux/775 ; DOI : https://doi.org/ 10.4000/recherchestravaux.775 


\section{Un théâtre qui exalte le vivre : Nathalie Papin, Dominique Richard, Betty Heurtebise, Joël Pommerat}

Certains ont eu des lieux de méditation, des rituels, des mythes, comme formes et espaces de recueillement. Nous pouvons peut-être avoir des lieux de théâtre. Des lieux où même si les quêtes aujourd'hui paraissent absurdes, vides, parce qu'on a perdu l'origine des mondes, on entend toujours un appel, sans savoir d'où.

C. RÉGY

Le théâtre devient un lieu de recueillement quand il est capable, comme le formule Enzo Corman, de «mettre à jour ces dimensions a priori cachées, pour ne pas dire secrètes, d'un réel à de maints égards inapprivoisables et malgré tout vécu ${ }^{\mathrm{I}}$ », quand il arrive à nous rappeler ce que nous partageons tous : la sensibilité débordante de l'enfance. Rares sont aujourd'hui les auteurs et metteurs en scène qui puissent nous accompagner vers ces sensations. Mais certaines écritures adressées aux plus jeunes semblent détenir ce pouvoir.

Partant des définitions de l'enfance proposées par Pierre Péju et JeanFrançois Lyotard, à savoir cet état que nous avons tous traversé "porteur de mouvement, de changement, qu'il soit pure croissance, plein de vie, de départs, de fuites, d'inquiétude, de menace, d'inventions sauvages, de grande

I. E. Corman, Ce que seul le théâtre peut dire (Considérations poélitiques), Besançon, Les Solitaires Intempestifs, 20I2, p. 68. 
innocence et de petite cruauté [...] capables de troubler ou d'infléchir notre façon d'être au monde ${ }^{2}$ » en les croisant avec des visions du théâtre développées notamment par Antonin Artaud et Claude Régy, il s'agira de montrer que la force de ces écritures n'est pas de nous faire retomber en enfance mais bien de nous mettre dans les conditions grâce auxquelles l'enfance se produit, de nous installer face à ce «devenir soi» qui nous accompagne tout au long de notre vie. Nous verrons que les écritures de Nathalie Papin et de Dominique Richard, les mises en scènes de Betty Heurtebise et de Joël Pommerat nous engagent tous, adultes comme enfants, dans des voyages intimes et collectifs qui nous permettent d'atteindre un en deçà ou un au-delà de la vie, ouvrant ainsi la voie à un théâtre philosophique.

\section{Des écritures qui révèlent la puissance de la vie}

\section{Saisir la vie par l'image de la mort}

La récurrence de la thématique de la mort dans les œuvres de Nathalie Papin et de Dominique Richard nous conduit à envisager un lien entre leurs écritures et une part des esthétiques théâtrales radicales comme celles d'Antonin Artaud, de Tadeusz Kantor, de Maurice Maeterlinck et de Claude Régy. Les pensées sur le théâtre de ces artistes se rejoignent en effet pour énoncer que «la vie ne peut être exprimée en art que par le manque de vie et le recours à la mort ${ }^{3}$ ». La mort serait une clé pour la compréhension du lien entre la vie et le théâtre.

La pièce la plus instructive à ce sujet est Debout ${ }^{4}$ de Nathalie Papin qui figure dans la liste des lectures proposée aux collégiens par le ministère de l'Éducation nationale. L'histoire débute par l'évocation directe de la mort et du suicide chez l'enfant.

Un cimetière, une tombe, un tas de terre près de la tombe, un fossoyeur et un enfant. Le fossoyeur découvre l'enfant allongé dans le fond du trou.

VICTOR Qu'est-ce que tu fais là?

L'enfant J'essaye de mourir's.

Comme une ritournelle, cet échange réapparaitra à l'identique entre le fossoyeur et l'enfant au début de la troisième scène : l'enfant se retrouve de nouveau allongé dans ce trou pour attendre la mort. À partir de cette image de la mort

2. P. Péju, Le goût pour l'enfance, Paris, Mercure de France, 20I4, p. 7.

3. T. Kantor, Le théâtre de la mort, Lausanne, L'Âge d'homme, 1977, p. 221.

4. N. Papin, Debout, Paris, L'École des Loisirs, coll. "Théâtre», 2000.

5. N. Papin, ibid., p. I3. 
symbolisée par cet enfant couché dans un cimetière, Nathalie Papin développe un véritable hymne à la vie. Le fossoyeur, celui qui introduit les défunts dans le monde des morts, va introduire l'enfant dans la vie en le conduisant dans le cimetière des Gitans, où les tombes sont comme des chapelles en verre sur lesquelles les fleurs, les objets et les photos abondent. L'auteure abolit complètement la frontière entre le monde des morts et celui des vivants, bouscule les représentations de la mort qu'a notre civilisation. Debout, le personnage principal, pénètre dans le cimetière des Gitans pour y rencontrer une ribambelle de mères toutes plus symboliques les unes que les autres : de la Mère Décaèdre aux bras et aux mois multiples à la Mère Araignée qui lui donnera des ailes, en passant par la Mère Jardin qui lui permettra de planter ses racines pour arriver à la Mère des Mères, Debout se nourrira au fil de la pièce de ces rencontres avec l'altérité, non pas pour trouver ce qu'il cherche mais, à force de chercher, pour trouver ce qu'il veut être. Il partira à Madagascar pour travailler la soie et le soi.

Dans L'ordre des morts, Claude Régy, s'appuyant sur des écrits de Rimbaud, nous rappelle la place de la mort dans l'enfance :

La force même du désespoir restitue, semble-t-il, certaines situations de l'enfance où la mort apparaissait si claire et comme une déroute, un jet continu'.

Nathalie Papin réussit, à travers Debout et son histoire, à approcher la sensibilité accrue que détient l'enfant. C'est à travers l'image de la mort qu'elle saisit l'essence de la vie. Il y a bien dans son écriture comme dans celle de Dominique Richard (en particulier dans la courte pièce Une journée de Paul ${ }^{7}$ ) une influence de la philosophie nietzschéenne parce que ces œuvres font jaillir le dionysiaque, par la construction et la destruction concomitantes, et l'orgiasme, un sentiment débordant de vie et de force au sein duquel même la douleur a encore l'effet d'un stimulant. Catherine Naugrette souligne dans le chapitre "Artaud, le théâtre et la vie" de son ouvrage L'esthétique théâtrale ${ }^{8}$ qu'il faut selon lui affronter la vie dans sa totalité : c'est un oui à la vie dans tout ce qu'elle porte à la fois de joyeux et de catastrophique. Le théâtre ne doit pas délivrer, comme le voulait Aristote, de la terreur et de la pitié, mais c'est en les traversant qu'il permet à l'homme d'être la joie du devenir. Dominique Richard qualifie son propre univers de "tragique joyeux ${ }^{9}$ ".

6. C. Régy citant Rimbaud, Les Poètes de sept ans dans L'ordre des morts, Besançon, Les Solitaires Intempestifs, 1999, p. 86-87.

7. D. Richard, Une journée de Paul, dans Théatre en court 2, Montreuil, Éditions Théâtrales jeunesse, 2007.

8. C. Naugrette, L'esthétique théâtrale (2 éd.), Paris, Armand Colin, coll. "Cursus», 2010.

9. Voir son mot d'auteur dans l'ouvrage de M. Bernanoce, Vers un théâtre contagieux, ouvr. cité, p. 44I-442, intitulé «Écrire par bouffée d'enfance : un tragique joyeux». 
La naissance de soi est tragique parce qu'elle est ponctuée de séparations, de renoncements, de différenciations. Une naissance passe forcément par un rejet. Comme un arbre et ses branches qu'il faut couper pour qu'il continue à vivre ${ }^{10}$.

Betty Heurtebise, directrice artistique de la compagnie bordelaise La Petite Fabrique, choisit de mettre en scène en 2012 la pièce de Nathalie Papin Le Pays de Rien ${ }^{\text {II }}$. Et c'est la libération des forces noires nécessaire pour vivre que vise sa mise en scène. La pièce raconte l'enfermement d'une jeune fille par son père tyrannique dans un pays où règne le Rien. C'est l'arrivée d'un jeune étranger qui lui révélera l'existence d'un ailleurs, la possibilité de vivre autrement. Dans la scène v, Nathalie Papin réveille la mémoire de la mort, de ce qui a disparu chez la Fille du roi en convoquant des squelettes amenés par un instrument de musique : la flûte. C'est le jeune étranger qui en donnant une flûte à la jeune fille va faire jaillir une force de vie. Cette flûte, réalisée avec la côte d'une petite fille qui riait toujours, a le pouvoir de donner du courage aux enfants qui deviendront squelettes s'ils ne trouvent pas d'espace pour poser leurs rêves.

Elle ramasse la flûte et souffle dedans. Des squelettes envahissent le pays de Rien. Ils dansent, ils sont drôles et joyeux ${ }^{12}$.

La flûte utilisée dans le spectacle de Betty Heurtebise a été fabriquée à partir d'un os d'animal. La Fille du roi, en soufflant dans cette flute, entre dans une transe proche de la sorcellerie. Son corps s'agite et semble possédé, révélant des mouvements secrets et contradictoires, comme si la mémoire de la mort libérait toutes les émotions enfermées par son père. Ce moment de danse crée une rupture avec le rythme global du spectacle tant au niveau sonore avec l'apport de sons de percussions qu'au niveau du jeu. Les mouvements de la Fille du roi, la flûte (premier instrument de musique utilisé par les hommes) nous renvoient à nos origines, à nos instincts sauvages. Nous pouvons alors penser au théâtre de la cruauté tel que le définit Antonin Artaud : un théâtre qui recouvre les origines dionysiaques et tragiques de la vie. Antonin Artaud entend le mot cruauté dans un sens large comme un appétit de la vie. Il me semble que Betty Heurtebise en choisissant de ne pas représenter les squelettes mais en les convoquant de manière virtuelle (projections sur le plateau) et par l'intermédiaire de cette flûte réalisée à partir d'un os d'animal rejoint pour partie la définition d'Antonin Artaud dans son deuxième manifeste du Théâtre de la Cruauté quand il souligne l'importance de créer un chevauchement entre les

Io. D. Richard, entretien avec A. Armellini, mai 2013.

II. Des images du spectacle sont disponibles sur le site Théâtre contemporain <http://www. theatre-video.net/video/Le-Pays-de-Rien-de-Nathalie-Papin-m-e-s-Betty-Heurtebise-extraits>. I2. N. Papin, Le Pays de Rien, Paris, L'École des loisirs, coll. "Théâtre», 2002, p. 27. 
différents langages scéniques : images, corps, lumières, sons... pour créer globalement un langage autre, peut-être capable d'aller chercher au fond de notre esprit des images peu ou pas utilisées. Le spectacle agit alors, me semble-t-il, comme une force qui révèle la puissance créatrice de la vie, qui exalte la vie; il réussit à bousculer le repos des sens, libérer l'inconscient opprimé, pousser à une sorte de révolte virtuelle.

\section{Briser le langage pour s'approcher du vivant}

\section{Comme l'écrivait Artaud,}

Briser le langage pour toucher la vie, c'est faire et refaire le théâtre [...]. Ceci amène à rejeter les limitations habituelles de l'homme et des pouvoirs de l'homme, et à rendre infinies les frontières de ce qu'on appelle la réalitéris.

Suivant le philosophe Paul Audir ${ }^{14}$ faisant référence à Jean-François Lyotard, l'enfance se situe dans un entre-deux, entre le fait d'être vivant et le moment où nous n'avons pas encore accès au langage, moment où nous sommes incapables de répondre. Tout au long de notre existence, nous continuons à porter cette incapacité et cette absence de sens qui peuvent resurgir à n’importe quel moment pour nous détourner de la signification, nous donner accès à un au-delà.

Le Garçon de passage de Dominique Richard me semble à la recherche d'un tel retour aux sources.

Revenons à l'étymologie du mot enfance - infans, qui ne parle pas. Cette étymologie est souvent reprise par les philosophes pour nous rappeler que le langage nous permet de maîtriser ce qui nous arrive. Dès lors que nous employons un mot, nous prenons une distance par rapport aux événements. La sortie de l'enfance s'accompagne de la maîtrise du langage. Et le discours est bien ce qui nous éloigne de l'état de sauvagerie de l'enfance.

D'ailleurs Jean-François Lyotard dans Lectures d'enfance ${ }^{\text {Is }}$ dit simplement que ces deux enfances (celle de l'enfant et celle de l'adulte) ont en commun d'être sans paroles (in-fantia). De même que l'enfant d'abord ne parle pas, l'enfance du discours est silencieuse.

Dominique Richard, dans chacune des pièces Les Saisons de Rosemarie, Hubert au miroir et Le Garçon de passage, publiées aux Éditions Théâtrales

13. A. Artaud, Le théatre et son double, Paris, Gallimard, 1964, p. 19.

I4. Les nouveaux chemins de la connaissance, Adieu l'enfance: Lyotard, l'enfance n'a pas d'àge, émission diffusée sur France Culture, le i6 décembre 2014.

I5. J.-F. Lyotard, Lectures d'enfance, Paris, Galilée, coll. "Débats», I99I. 
Jeunesse, s'amuse avec le langage jusqu'à l'invention de nouvelles langues. Il parvient ainsi à nous rappeler la dualité du langage à la fois utile et aliénant.

Dans Le Garçon de passage, l'auteur invente une langue, le "pallakch ", pour raconter la relation entre trois enfants sur une île mystérieuse. Une journée sur cette île leur permet de traverser de nombreux questionnements : grandir, désirer, découvrir, vivre, regarder naître les identités... Il est intéressant de lire la note écrite par l'auteur, à la fin de la pièce. Dominique Richard y définit ce langage :

[...] une langue inventée, langue du secret, une langue d'avant, langue de la dissolution ou de la dissémination, le contraire du discours possible ou d'une parole qui se donne $[\ldots]$ où dans un seul mot sont inscrits, enveloppés, tous les possibles [...] le plus simple pour progresser est encore d'écouter la source et le tonnerre, de se laisser aller au surgissement, en soi, de l'évidence des mondes [...] Le pallakch est la seule vraie langue, celle des mondes et de ses bruits, celle des tripes et de ses souffles. Les autres n'en sont que de grossières approximations. [...] Nous avons tous commencé à parler le pallakch mais bizarrement en grandissant, nous l'avons oubliér ${ }^{16}$.

Outre cette note édifiante, les personnages donnent des explications à propos de cette langue inventée que le garçon de passage essaie de comprendre pour entrer dans le clan formé par la fille et le garçon. De nouveau surgit l'idée de renaissance dans la mort que la langue pallackh dit par le mot «bo» qui signifie "vivre-mourir".

Cette recherche autour du langage peut alors nous ramener aux expériences d'Antonin Artaud qui s'est intéressé à un langage primitif, pulsionnel, comme celui de l'enfant peut-être. Poussant le langage jusqu'aux limites de l'articulation, Artaud interroge le mot et son pouvoir de référence; il fait sortir le langage du schéma signifiant-signifié pour aller vers l'expulsion de mots. Dominique Richard, d'une façon proche, travaille le langage pour le libérer, pour le faire sortir de son emprisonnement dans la signification en inventant le "pallakch». Cette libération des mots pourrait nous permettre de retrouver la culture défendue par Antonin Artaud, celle liée à la vie sauvage, laissant s'échapper la magie de la vie.

En convoquant la mort ou en brisant le langage, ces artistes ouvrent des portes vers des espaces inconnus, non maîtrisés et maîtrisables. Ils sont des «résistants», au sens que Gilles Deleuze donne au mot «résistance» dans la définition que propose son abécédaire : l'artiste «libère la vie que l'homme a emprisonnée ${ }^{17}{ }^{\prime}$. On peut ajouter qu'il exalte la vie, qu'il capte l'Enfantin

I6. D. Richard, Le Garçon de passage, Montreuil, Éditions Théâtrales Jeunesse, 2009, p. 75-77.

I7. G. Deleuze dans L'Abécédaire de Gilles Deleuze, produit et réalisé par P. A. Boutang, Paris, Éditions Montparnasse, DVD, 2004. 
quand «il se manifeste par sensations et clartés soudaines, par blocs sonores ou saveurs demeurés intactes ${ }^{18}$ ", comme l'écrit Pierre Péju, et qu'il parvient à le transmettre pour renouveler nos perceptions des choses et de nous-mêmes.

\section{Vers l'expérimentation d'un réel élargi}

Michel Foucault, dans une conférence radiophonique de 1966 qui a donné naissance à un texte publié aux Éditions Lignes, Le corps utopique, arpente le corps comme un territoire, un espace limité, auquel chacun de nous est condamné, et que nous partageons. L'exploration du corps par Michel Foucault est finalement assez proche de celle des enfants. Les auteurs et les metteurs en scène de notre étude jouent avec l'exploration du corps : jeux de miroir, dédoublement, disparition, apparition... Si les expériences autour du corps, du double notamment, sont surtout marquées dans notre civilisation par l'angoisse, elles peuvent aussi être l'occasion de se promener dans des contrées lointaines et enchantées, nous transporter ailleurs, être le support de métamorphoses nous invitant à découvrir des dimensions cachées de notre existence, à atténuer les frontières entre le réel et l'imaginaire. Relisons Foucault :

Corps incompréhensible, corps pénétrable et opaque, corps ouvert et fermé: corps utopique. Corps absolument visible, en un sens [...]; pourtant, ce même corps qui est si visible, il est retiré, il est capté par une sorte d'invisibilité de laquelle jamais je ne peux le détacher. Le corps, fantôme qui n’apparaît qu'au mirage des miroirs. [...] Est-ce que vraiment j'ai besoin des génies et des fées, et de la mort et de l'âme, pour être à la fois indissociablement visible et invisible? [...] Il court, il agit, il vit, il désire, il se laisse traverser sans résistance par toutes mes intentions. Hé oui ${ }^{19}$ !

\section{La découverte de soi par l'exploration du corps}

Dans la pièce de Dominique Richard, Les Saisons de Rosemarie, Rosemarie est une petite fille dans la confusion : elle cherche qui est, elle s'explore. Dès la première séquence, Dominique Richard fait apparaître à ses côtés «le garçon " que Rosemarie invitera très vite à mettre ses pas dans les siens pour la suivre et sautiller avec elle dans les flaques. Ce double masculin a quelques traits singuliers. Il s'oppose à Rosemarie dans sa manière de communiquer. Ses phrases sont très longues, il utilise un mot pour un autre alors que Rosemarie

I8. P. Péju, Le goût pour l'enfance, ouvr. cité, p. Io.

19. M. Foucault, Le corps utopique, les hétérotopies, Paris, Nouvelles Éditions Lignes, 2009, p. I3, I4. 
s'exprime le moins possible. De plus, il remet très souvent en question les règles imposées par le monde des adultes :

«Je me demande si les adultes n'essaient pas de nous enseigner les choses qu'on connaît pour qu'on n'arrive plus à les faire ${ }^{20}$."

Il est un détour pour permettre à Rosemarie de mieux se découvrir, faire l'expérience de soi, s'amuser avec ses propres limites. Dans une séquence intitulée "Jeu», Rosemarie change de corps et s'installe dans celui du garçon qui lui rend la pareille.

«J'aimerais bien savoir qui je suis. En me rencontrant, je pourrai mieux m’observer et m'espionner.

Ça fait un peu peur de ne plus être soi-même. Et si je ne pouvais plus me retrouver après le jeu ${ }^{21}$ ?»

Rosemarie va ainsi pendant la pièce faire un voyage avec son double masculin et puis l'abandonner progressivement pour laisser éclore cet autre qu'elle enfermait à l'intérieur d'elle-même. Le monologue de fin est éloquent. Elle est seule et se sent enfin exister dans sa tête et dans son corps.

Cette capacité d'être soi et plusieurs autres est une préoccupation récurrente dans les ouvres de Dominique Richard dont l'écriture ne cesse de réfléchir à la construction de l'identité de l'enfant, à la naissance du «moi».

Dans Hubert au miroir, comme le titre l'indique, c'est dans l'expérience du miroir et du reflet qu'Hubert se découvre au fil de la pièce. Dominique Richard s'exprime à ce sujet dans la note publiée à la fin de la pièce.

Le sujet que nous sommes reste une énigme, redécouvrant sans cesse ce qui le constitue, être multiple et provisoire, en devenir, reprenant le fil de sa vie à mesure qu'elle s'écoule... Hubert explore ses propres labyrinthes, à la recherche du secret qui le constitue : labyrinthes des corps, de miroir en miroir, labyrinthe de la pensée, d'énigme en énigme, pour ne trouver que le vide en lui-même, l'absence qui le hante et dont il va falloir faire le deuil pour grandir ${ }^{22} \ldots$

Dans Mange-moi, Nathalie Papin raconte l'histoire d'Alia aux prises avec son corps obèse qui lui vaut les moqueries de ses camarades. Elle cherche à disparaître, à se débarrasser de son corps encombrant.

«Vous ne rirez pas longtemps quand j'aurai disparu, et que personne ne saura me retrouver ${ }^{23}$.»

20. D. Richard, Les Saisons de Rosemarie, Montreuil, Éditions Théâtrales Jeunesse, 2004, p. 39.

2I. Ibid.

22. D. Richard Hubert au miroir, Montreuil, Éditions Théâtrales Jeunesse, 2008, p. 93-94.

23. N. Papin, Mange-moi, Paris, L'École des loisirs, coll. "Théâtre», p. 9. 
Dans sa course pour échapper aux autres qui lui rappellent sans cesse ce corps insupportable, elle trébuche, s'assied devant un horizon et rencontre un ogre mangeur d'horizon qui va lui ouvrir son ventre pour qu'elle vienne s’y réfugier. À partir de là, Alia voyage à l'intérieur du ventre de l'ogre où elle rencontrera un dévoreur de livres, un mangeur de mémoires et surtout un oiseau étrange qui lui confiera la mission de sauver l'ogre. Nathalie Papin crée des lieux autres, des lieux fictifs hautement symboliques qui rapprochent son écriture de celle des symbolistes tels que Maurice Maeterlinck. Alia (dont le sens étymologique est l'"autre») voyage à l'intérieur du corps d'un autre, l'ogre, puis sur une ligne d'horizon. Ces espaces - le ventre vide de l'ogre, les côtes de l'ogre, l'horizon; ces personnages croisés : le dévoreur de livres, le mangeur de mémoire, l'oiseau, l'ogre «anogrexique» transportent Alia entre les deux mondes du visible et de l'invisible, là où personne ne la trouvera. Ce voyage secret lui permet de se métamorphoser, de changer ses perceptions, de devenir toute neuve, de libérer son chagrin.

\section{Des créateurs d'espace mental}

Dans Le corps utopique, Michel Foucault développe le concept d'hétérotopies, ces lieux dans lesquels nous percevons différemment la réalité ainsi que notre propre identité. Ils sont des espaces mythiques ou réels, mais des espaces de contestation de l'espace dans lequel nous vivons. Certains auteurs et metteurs en scène pour la jeunesse font naître ces espaces où les frontières entre le réel et l'imaginaire deviennent floues, voire inexistantes.

Betty Heurtebise, dans sa mise en scène du Pays de Rien, explore la force de l'obscurité, ou comment voir ce que l'œil ne voit pas. Elle joue avec la notion de double et propose une nouvelle lecture dramaturgique de la pièce de Nathalie Papin. Par un travail sur la lumière qui révèle une présence fantomatique, l'existence réelle du jeune garçon est questionnée. Comme le souligne Claude Régy dans L'état d'incertitude, c'est l'obscurité qui est fondatrice parce qu'elle donne naissance à un certain jour artificiel.

Ce n'est pas vrai qu'on ne voit pas un être vivant s'il reste dans l'ombre. On ressent comme davantage sa présence, davantage de la force invisible qu'un centre en lui irradie. On sent mieux non pas ce qu'il est mais ce qu'il aurait pu être. Et bien sûr prédomine ce qu'aurait pu être sur ce qui est. Il ne faut donc pas montrer la lumière de ce qui est, mais rester dans la lumière infinie de ce qui serait possible, hors des limites du temps ${ }^{24}$.

24. C. Régy, L'état d'incertitude, Besançon, Les Solitaires Intempestifs, 2002, p. IOI. 
L'arrivée du jeune garçon sur le plateau devant la jeune fille crée un faceà-face saisissant entre les deux corps, celui du garçon dans l'obscurité et celui de la fille dans la lumière. Le garçon s'avance et la Fille du roi recule comme effrayée par quelque chose qui surgirait d'un ailleurs qu'elle ne connaît pas, d'elle-même peut-être. Le jeune garçon semble plus présent dans l'ombre qu'en pleine lumière, comme si sa présence irradiait l'espace pour dévoiler de nouveaux espaces dans le corps de la jeune fille.

C'est à travers cet invisible, qu'une sur-présence s'installe, à travers cette absence corporelle on pourrait deviner l'inconnaissable, l'altérité absolue, tout peut venir s'y inscrire ${ }^{25}$.

Interrogé par Joëlle Gayot sur son théâtre, Joël Pommerat ${ }^{26}$ insiste sur le fait que la fiction est un détour pour mieux faire l'expérience du réel. Son théâtre est un chevauchement entre les différents langages : parole, son, lumière qui créent un espace ouvrant les champs de la perception et de l'imagination. Quand on l'interroge sur l'obscurité de ses spectacles, il prend l'exemple du personnage d'un roman que nous sommes en train de lire, ce personnage avec qui nous finissons par établir une grande proximité mais dont la forme précise du corps nous échappe. Plusieurs impressions, plusieurs visages, plusieurs corps parfois contradictoires se dessinent. Différents éléments se superposent pour composer un être à la fois vrai et multiforme. C'est peut-être cette perception qui rend finalement la juste complexité de la relation que nous entretenons avec le monde qui nous entoure, floue et ambiguë.

Thomas Boccon-Gibod ${ }^{27}$, dans son article sur le théâtre de Joël Pommerat, développe sa proximité avec les réflexions de Michel Foucault sur la fiction et les hétérotopies. Les mises en scène de Joël Pommerat ont cette capacité d'attirer notre œil et notre oreille vers des choses qui paraissent ordinaires mais qui, en créant des espaces qui étendent nos zones de perceptions, transforment le plateau de théâtre en lieu de philosophie concrète.

Les théâtres de Joël Pommerat, de Betty Heurtebise et de Claude Régy bousculent nos habitudes perceptives. Ces auteurs travaillent à la recherche d'une ultra-sensibilité, d'une hyper-lucidité qui fait percevoir, entendre, ressentir un détail de la façon la plus aiguë. Il s'agit, dans leur travail, de révéler, réveiller, afin de nous restituer une étrangeté que l'habitude nous fait perdre, cette étrangeté

25. C. Régy, L'ordre des morts, Besançon, Les Solitaires Intempestifs, I999, p. 83.

26. J. Pommerat, Joël Pommerat, troubles, entretiens avec Joëlle Gayot, Arles, Actes Sud, 2009.

27. T. Boccon-Gibod, "Je tremble et Cercles / Fictions : l'utopie théâtrale de Joël Pommerat, une politique de l'imaginaire", Agôn [en ligne], Horizons politiques de la communauté, nº 3 : Utopies de la scène, scènes de l'utopie, [Dossiers, mis à jour le : I9/oI/20II], <http://agon. ens-lyon.fr/index.php?id=I56I > . 
qui vient de nous, cette étrangeté qui est peut-être la part d'enfance perdue que nous cherchons. Ils atteignent ainsi un espace polymorphe et polysémique qui ne transpose pas les apparences du monde visible, mais s'offre plutôt comme une voie ouverte à notre imaginaire.

Tous, me semble-t-il, cherchent à montrer moins pour suggérer plus, pour ouvrir l'œuvre à de multiples perspectives. Ils parviennent à stimuler les sens du spectateur pour développer un voir qui va au-delà de la fonction visuelle, un entendre qui va au-delà de la fonction auditive et ouvrent des champs vers des horizons sans limite. Comme l'écrivait Maeterlinck : "Il s'agirait plutôt de faire voir ce qu'il y a d'étonnant dans le fait seul de vivre ${ }^{28}$.»

\section{Des penseurs de l'invisible}

Ces artistes ouvrent ainsi la porte de préoccupations complexes qui nous constituent tous et qui concernent la mort, le temps, l'être, l'origine... Leurs écritures et leurs mises en scène modifient notre rapport au monde en nous éloignant des représentations concrètes, des perceptions brutes. Elles créent ainsi des espaces de rêverie, d'obscurité et de silence dans lesquels la réflexion peut s'installer. Les enfants, petits philosophes qui s'ignorent, s'y installeront en premier.

Pour terminer, on peut qualifier ces artistes de penseurs de l'invisible car ils explorent les limites pour nous amener à réfléchir sur l'illusion, à découvrir ces dimensions secrètes du réel, qui étendent nos moyens de perception pour exalter le vivre et peut-être nous rapprocher de «notre véritée 9 ».

28. M. Maeterlinck, cité par S. Ballestra-Puech, dans "Le Tragique au quotidien », Le Trésor des Humbles, Paris, Mercure de France, I896, p. I6I.

29. M. Foucault, Le courage de la vérité. Le gouvernement de soi et des autres II. Cours au Collège de France (1984), Paris, Éditions de l'École des hautes études en sciences sociales - Gallimard Seuil, coll. «Hautes Études», 2009, p. I3-I4. 